\title{
Evaluation and management of facial granuloma caused by various injection materials
}

Jin Woo Jang ${ }^{1}$, Sang Yoon Kang ${ }^{1,2}$

${ }^{1}$ Department of Plastic Surgery, Kyung Hee University Hospital, Seoul;

${ }^{2}$ Department of Plastic Surgery, Kyung Hee University College of Medicine, Seoul, Korea

\begin{abstract}
Background: Various substances are currently being used for cosmetic or postsurgical reconstruction reasons. Injecting of various materials into the face may provoke inflammatory or granulomatous reactions, infection and tissue necrosis. Among these reactions are foreign body granulomas. This study aims to describe the clinical characteristics of and therapeutic approaches used in patients with facial foreign body granulomas caused by the injection of various substances.

Methods: From 2007 to 2020, a total of 64 patients visited our hospital due to inflammatory signs, palpable masses or surface irregularities. We reviewed patient characteristics, type of injected material, medical history and treatment outcomes. The treatment results were scored with patient satisfaction and statistical analysis of the treatment period was performed.

Results: Most patients underwent conservative treatment followed by surgical treatment because of persistent symptoms; one patient did not require surgery. All patients reported good treatment results and satisfaction. The earlier the surgical treatment was performed, the shorter the treatment period and the higher patient satisfaction scoring.

Conclusion: Granulomatous changes to the face are an emerging concern in various cosmetic procedures and surgeries, including fillers, silicone implants and autologous fat injections. Our findings strongly suggest that patient symptoms require accurate diagnosis and surgical treatment.
\end{abstract}

Keywords: Fat / Granuloma, foreign body / Inflammation

\section{INTRODUCTION}

Skin contouring for cosmetic or postsurgical reconstruction reasons is growing in popularity. Various substances are currently being injected for these purposes. Because of the short recovery period, fillers and autologous fat injection (AFI) are becoming more common. In facial contouring surgery using AFI, repetitive injections incorporating frozen fat are widely used due to their convenience [1]. Meanwhile, although some research suggested that indiscriminate injections of substances

\footnotetext{
Correspondence: Sang Yoon Kang

Department of Plastic Surgery, Kyung Hee University Hospital, Kyung Hee

University College of Medicine, 23 Kyungheedae-ro, Dongdaemun-gu, Seoul

02447, Korea

E-mail: nuelk@nate.com
}

Received November 24, 2020 / Revised February 15, 2021 / Accepted February 15, 2021 other than medical fillers by unlicensed offices for cosmetic purposes are waning, they continue to be performed. The side effects of these unsanctioned procedures are serious.

Injecting foreign substances into the superficial layers of the body can cause several kinds of delayed side effects such as swelling, pain, erythema, bleeding, acute inflammatory reactions. Moreover, it may provoke allergic reactions, inflammatory or granulomatous reactions, infections, and tissue necrosis [2]. As procedures performed in the face are increasing in popularity, complications are also on the rise. Among these complications are foreign body granulomas with inflammation.

On biopsy during inflammation treatment, foreign body granulomas composed of macrophages and foreign giant cells have been identified in patients who underwent AFI, filler injection, or implantation. Foreign body granulomas are chronic 
inflammatory reactions with various etiologies that appear as lesions comprising a collection of immune cells [3]. Multiple factors affect their etiology: the number of injections, the use of stable fat, modified fillers, or unknown substances; patient-specific elements, infection, and the complexity of the procedures.

This study describes the clinical characteristics of patients with facial foreign body granulomas caused by the injection of various substances and their treatment.

\section{METHODS}

From 2007 to 2020, a total of 64 patients visited our hospital due to inflammatory signs such as pain, swelling, or redness, or who complained of surface irregularities, palpable masses, fistula, or tenderness. All patients were diagnosed with facial foreign body granulomas on pathologic examination.

We reviewed each patient's age, sex, type of injection, injection site, complaints according to site, treatment history, injection material, treatment method, and posttreatment condition based on medical records and clinical pictures.

For patients with mild symptoms, conservative treatment included anti-inflammatory drugs, antibiotics, and/or hyaluronidase, followed by additional surgical treatment when necessary. Conversely, patients with severe acute symptoms were treated with surgical intervention first, and underwent emergent surgeries such as incision and drainage, controlling acute symptoms early to avoid further aggravation or extensive soft-tissue injuries.

Treatment results were evaluated using patient satisfaction surveys after treatment, either as a questionnaire at the outpa- tient clinic or by phone survey. The satisfaction questionnaire included five responses: very satisfied, satisfied, just so, dissatisfied, and very dissatisfied.

For statistical analyses, Spearman correlation analysis was performed to examine the associations between patient satisfaction and period from visit to surgical treatment. $p$-values $<0.05$ were considered statistically significant. Statistical analyses were performed using SPSS version 23.0 (IBM Corp., Armonk, NY, USA).

The study was approved by the Institutional Review Board of Kyung Hee University Hospital (IRB No. 2020-12-001) and performed in accordance with the principles of the Declaration of Helsinki. Patients provided written informed consent for publication and the use of their images.

\section{RESULTS}

In the patient's analysis, various information was analyzed and the types of injection materials, symptoms and examination modalities for diagnosis were evaluated (Table 1). The incidence rate of foreign body granuloma was highest among patients in the fifth decade of life (40.6\%). On analysis of the injection material and the site of occurrence, the most prevalent sites were the nose, forehead, glabella, and lower lip, respectively. Multiple sites were also confirmed in eight cases (Table 1).

Thirty-five patients were treated at other hospitals before visiting our hospital, albeit without significant improvement. $\mathrm{Pa}-$ tients with acute symptoms such as swelling, redness, or tenderness were initially managed with conservative treatment. Those included careful observation, cooling using an ice pack, and

Table 1. Injected site according to the procedure

\begin{tabular}{|c|c|c|c|c|c|c|}
\hline Site & $\begin{array}{l}\text { Autologous fat } \\
\text { injection }\end{array}$ & $\begin{array}{l}\text { Silicone implant for } \\
\text { augmentation }\end{array}$ & $\begin{array}{l}\text { Surgical implant } \\
\text { (thread, plate, etc.,) }\end{array}$ & Filler injection & $\begin{array}{l}\text { Unknown illegal } \\
\text { substance }\end{array}$ & Total \\
\hline Forehead & 2 & 1 & 3 & 4 & 3 & $13(20.31)$ \\
\hline Glabella & - & - & - & & 8 & $8(12.50)$ \\
\hline Nose & - & 5 & 1 & 4 & 5 & $15(23.43)$ \\
\hline Upper lip & - & - & - & - & 1 & $1(1.56)$ \\
\hline Lower lip & - & - & - & 3 & 3 & $6(9.37)$ \\
\hline Mentum & - & - & 2 & - & - & $2(3.12)$ \\
\hline Temple & - & - & 1 & - & - & $1(1.56)$ \\
\hline Upper lid & 1 & - & 1 & - & - & $2(3.12)$ \\
\hline Lower lid & 2 & - & - & - & 1 & $3(4.68)$ \\
\hline Nasolabial fold & - & - & - & - & 1 & $1(1.56)$ \\
\hline Ear & - & - & 1 & - & - & $1(1.56)$ \\
\hline Cheek & - & - & 1 & 1 & 1 & $3(4.68)$ \\
\hline Multiple site & 1 & 1 & 1 & 1 & 4 & $8(12.50)$ \\
\hline Total $(n=64)$ & $6(9.37)$ & 7 (10.93) & $11(17.18)$ & 13 (20.31) & 27 (42.18) & \\
\hline
\end{tabular}

Values are presented as number or number (\%). 
medications such as antibiotics, anti-inflammatory drugs, steroids, and intralesional injections. After inflammation had subsided with conservative management, surgical excision was performed. Patients with chronic symptoms such as a palpable mass or bulging were also treated with surgical excision. Six of the patients who visited our hospital underwent surgery on the same day as their initial visit, while 41 patients underwent conservative treatment for less than 1 month, followed by early surgery an average of 2 weeks later. Sixteen patients underwent surgery after conservative treatment for at least 1 month, with an average duration of 4.3 months. All patients underwent surgical treatment with incision and drainage or direct excision or foreign body removal except one.

Twenty-six patients underwent foreign body removal, followed by partial resection around the lesion and biopsy. More invasive surgical treatments such as curettage or excision were required if no improvement was observed despite treatment. Extensive surgical treatment was performed in 29 patients.

Table 2. Treatment modality

\begin{tabular}{lc}
\hline Variable & Total \\
\hline Conservative treatment & 1 \\
Surgical treatment & \\
Foreign body removal + partial resection & 26 \\
Extensive excision + curettage & 33 \\
Emergent incision + drainage & 4 \\
\hline
\end{tabular}

Values are presented as number.
Among them, seven patients were treated with artificial dermal transplantation to correct the deformities caused by extensive resection. Four patients underwent two or more surgical treatments. Emergent incision and drainage procedures were performed in four patients with discharge and tenderness (Table 2).

Several treatments were performed for the correction of possible deformities after foreign body removal surgery. In nine patients, immediate reconstruction with transplantation of artificial dermis was performed to prevent depression deformity. In two patients, deformities were corrected through upper blepharoplasty, and in one patient lower blepharoplasty. Steroid injection (triamcinolone) was used to correct irregular deformities in the majority of the rest of patients. In some cases, especially those with forehead lesions, mild compression was used along the uneven surface of the skin after surgical treatment. The process of diagnosis, treatment, and postoperative management were written as an algorithm and presented to show reproducibility (Fig. 1). Two cases were introduced and both patients ended follow-up with good treatment results (Figs. 2, 3).

The degree of satisfaction after surgery was classified into five categories from 5 points for "very satisfied" to 1 point for "very dissatisfied." The result was 3.98 points, which suggested that patients were generally satisfied with their results. More specifically, however, 12 people were "very satisfied," 43 people were "satisfied," four people responded “just so," and five people were

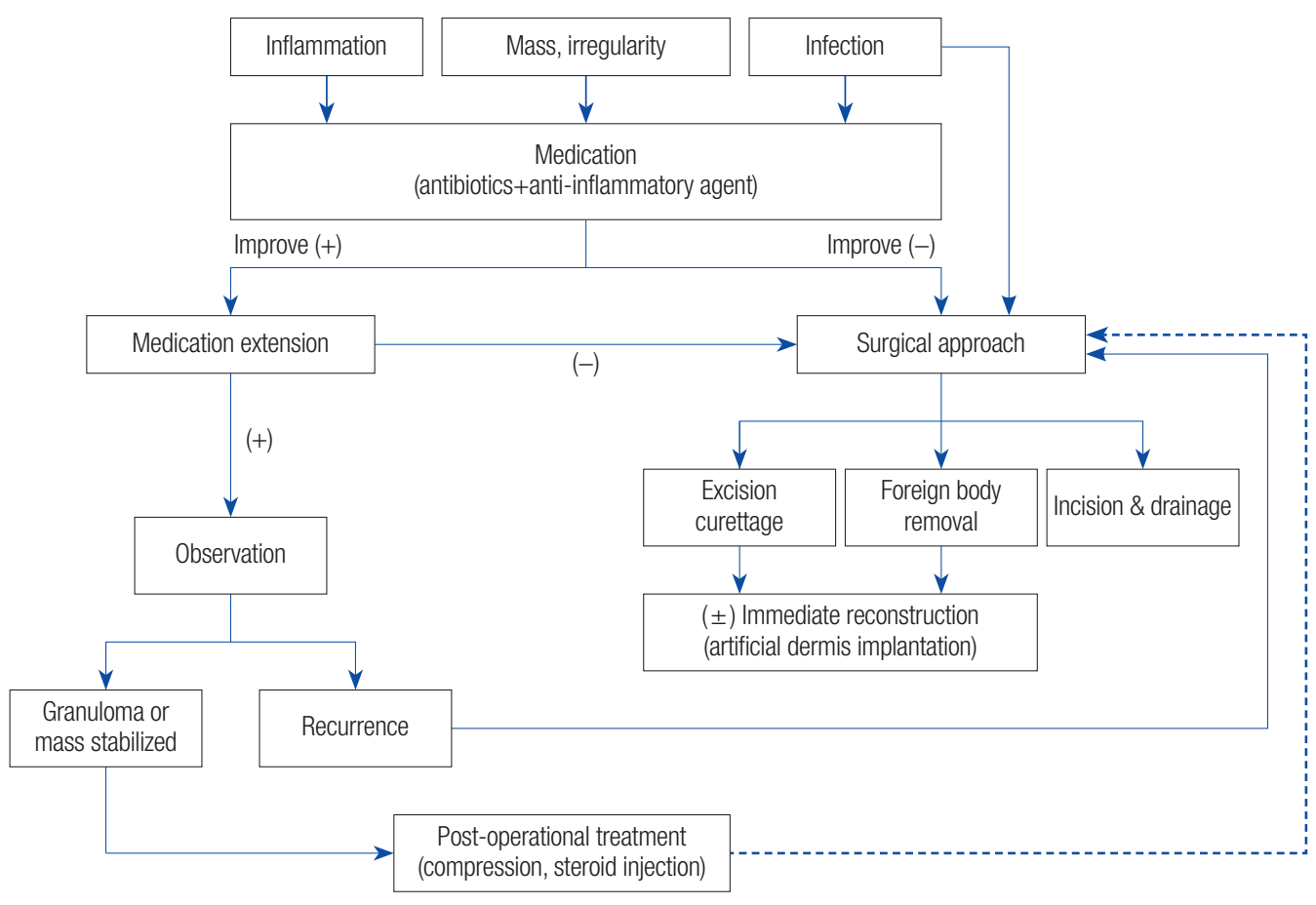

Fig. 1. An algorithm for evaluation and treatment of foreign body granuloma. Dotted line indicates the possibility of occurrence. 

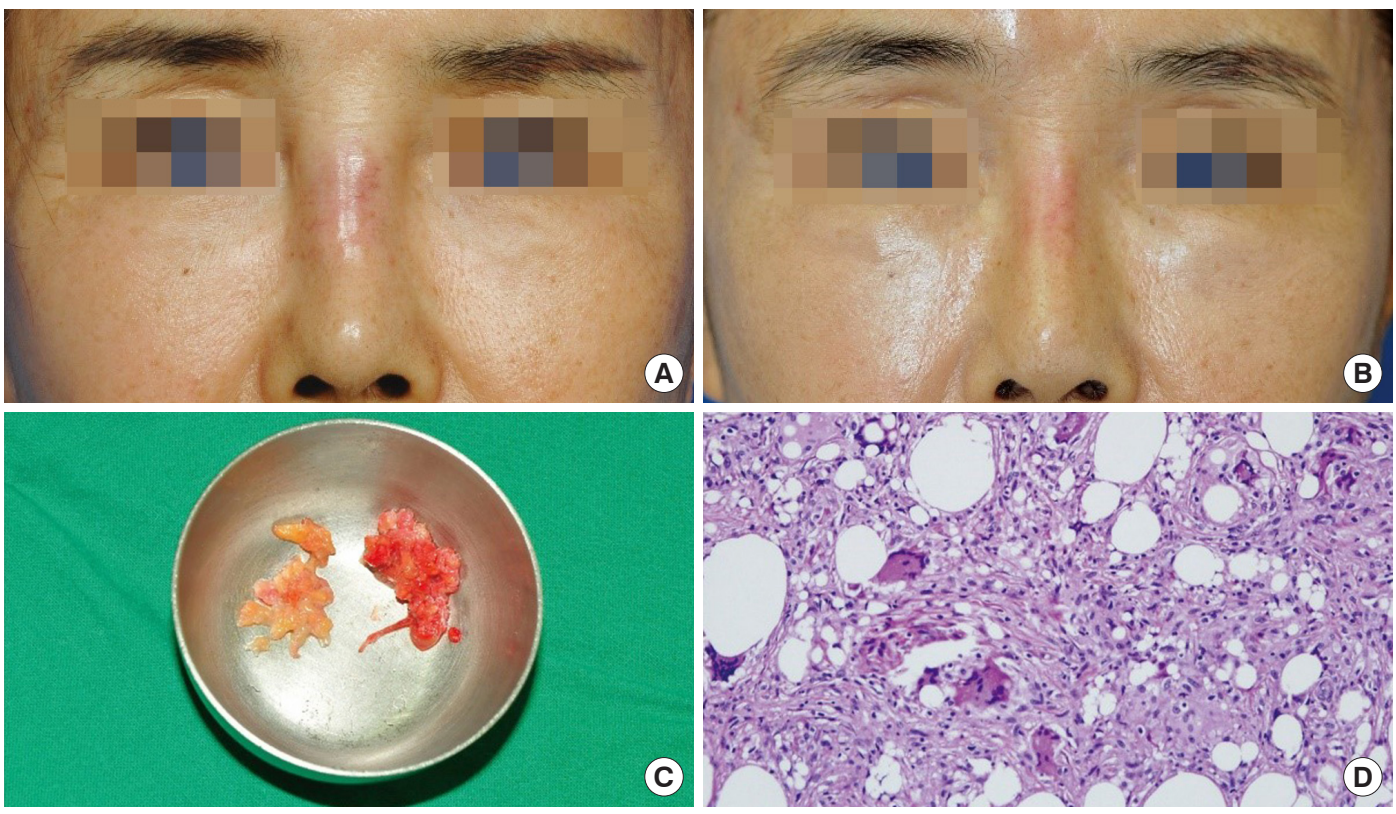

Fig. 2. (A) A 61-year-old woman complained of swelling, tenderness, and redness in her nasal dorsum near a silicone implant inserted 20 years prior. (B) She underwent curettage and excision of the suspicious lesion, then reconstruction with an allogeneic dermis graft following resolution of the inflammatory signs with antibiotics. Her symptoms did not recur after 1 year and she experienced no complications. (C, D) Biopsy results showed a foreign body granuloma (H\&E, $\times 400)$.
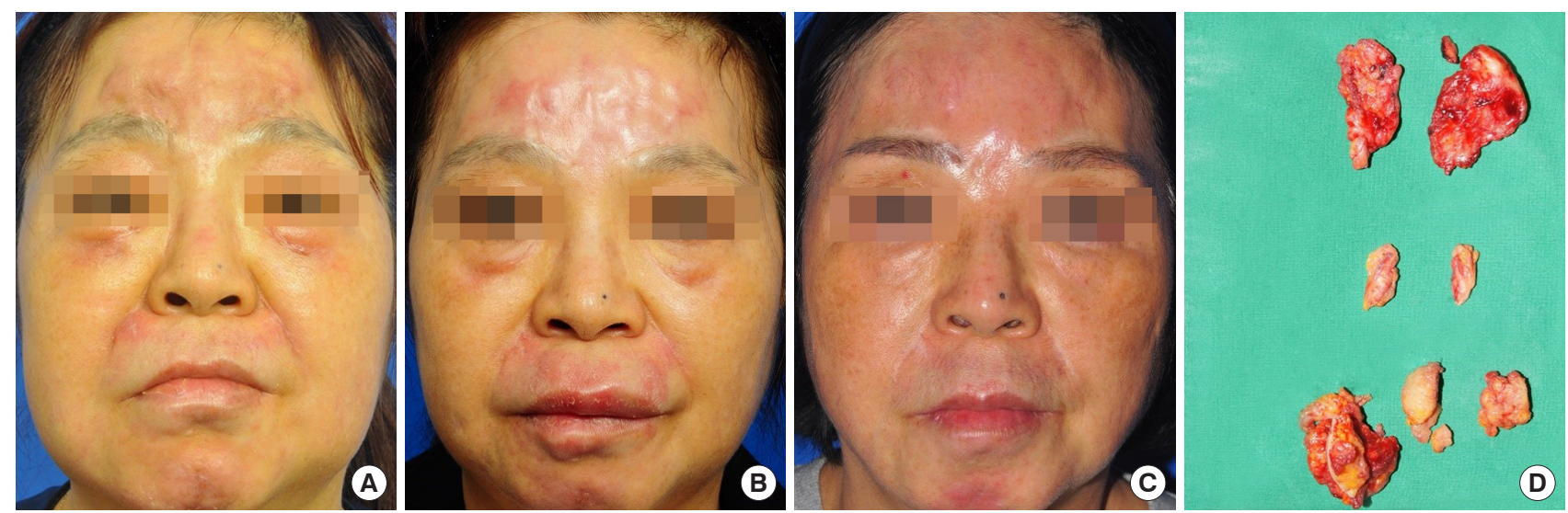

Fig. 3. (A) A 57-year-old woman complained of multiple areas of facial swelling and redness 2 years after injection of unknown material. (B) The patient complained of symptoms all over the face. After stabilization through conservative therapy for about 2 months, the foreign body was removed. (C) Conservative treatment without additional surgery is ongoing. (D) The foreign body had infiltrated into the tissue, and biopsy results confirmed a foreign body granuloma.

"dissatisfied." Those patients who were dissatisfied reported deformities and scarring from the surgery, with symptoms recurring intermittently in some cases. We performed additional surgery such as further resection of the foreign body and scar revision for resolving these issues.

On analysis of the correlation between patient satisfaction and the period from visitation to the date of surgical treatment (Table 3), the longer the period until surgical treatment, the lower the patient's satisfaction with treatment, suggesting a negative correlation between the two variables $(p<0.001)$. This in-
Table 3. Statistical analysis $(n=63)$

\begin{tabular}{lccccc}
\hline & \multicolumn{3}{c}{ Total } & Correlation & $p$-value ${ }^{\text {a) }}$ \\
\cline { 2 - 5 } & Mean & SD & Median & coefficient & \\
\hline Patient satisfaction & 3.98 & 0.81 & 3.96 & -0.7 & $<0.001$ \\
$\begin{array}{l}\text { Period from visit to surgical } \\
\text { treatment }\end{array}$ & 1.24 & 2.01 & 0.40 & & \\
\hline
\end{tabular}

a)Spearman correlation analysis.

dicates that foreign body reactions eventually require surgical treatment, and if surgical treatment is performed as soon as 
possible, the entire treatment period can be shortened and satisfaction can be increased.

\section{DISCUSSION}

Foreign body granuloma is a type of granulomatous tissue reaction that occurs in response to the presence of various substances. Chronic inflammatory cells infiltrate the foreign body injection site, resulting in a subcutaneous nodule and induration. Depending on the type, purity, and dose of the injected material, foreign body granulomas can be induced. Also, repeated injections can stimulate a large number of macrophages, leading to foreign body granulomas [4-6].

Histopathological findings are important in estimating the causative factor of foreign body granulomas. In foreign body reactions caused by the injection of paraffin and liquid silicone, a characteristic Swiss-cheese shape with circular or elliptical fat vacuoles is apparent. Multinuclear giant cells typically surround the lesion and chronic inflammatory cells infiltrate the interstitial tissue [7].

In the field of plastic surgery, various surgical procedures involve the injection of medical fillers with proven safety, including collagen or hyaluronic acid preparations [8,9]. Moreover, in the case of foreign body granulomas caused by medical filler injection, in addition to surgical resection, treatments using various approaches such as hyaluronic acid degrading enzyme injection, local or systemic steroid therapy, or treatment with bleomycin have been introduced $[9,10]$.

As fat grafting has become more popular, complications have also grown more common. Harvesting autologous fat using liposuction is a comfortable, repeatable, and low-cost procedure for most patients [11,12]. In 2009, a task force formed by the American Society of Plastic Surgeons assessed autologous fatgrafting techniques to determine their safety and efficacy [13] and reported that fat grafting is safe for various medical conditions, with a lower risk of com-plications than the risk levels recorded for other types of surgery. However, AFI's main obstacle in augmentation is an unpredictable outcome from a high absorption rate and necrosis at the grafted site [14]. For acute inflammation after fat grafting, good results can be obtained by re-transplanting fat after treatment for inflammation [15]. However, in this case of patients who visited the hospital due to persistent chronic inflammation, it was judged that the possibility of recurrence would be high if the fat was re-transplanted. This is because it was more difficult to completely remove the lesion, especially in patients with chronic lesions.

In addition, as mentioned earlier, the injection of illegal substances is still being carried out. Many people come to the hos- pital with chief complaints of deformity, symptoms, and complications caused by the injection of illegal substances. Indeed, $42 \%$ of the patients in this study did not know the type of substance they were injected with; thus, we did not know how and when the foreign body reaction mechanisms were triggered [16].

Doh et al. [17] reported a case of bioabsorbable plate-related foreign body granuloma in a patient with facial bone fracture. They suggested that caution be taken when using this substance in vivo. Inadequate degradation may cause delayed foreign body granulomatous changes. We have experienced two cases of plate-related lesions: one involved an absorbable plate used to fix the anterior wall of the maxilla through a gingivobuccal approach, while the other incorporated a titanium plate for frontal bone fixation. Foreign body granuloma should be suspected based on review of the previous surgical history concerning the patient's symptoms.

Using imaging modalities such as computed tomography (CT) or magnetic resonance imaging (MRI) to identify the lesion and surrounding area may be necessary to identify the cause of the symptoms and for surgical planning. Ko et al. [18] introduced high-resolution ultrasonography as a relatively inexpensive and useful diagnostic modality than CT or MRI.

In patients with severe inflammation, the use of antibiotics to reduce symptoms was attempted, with subsequent surgical resection. If nonsurgical treatment fails, and inflammation, pain, and paresthesia are observed, surgical treatment is performed. Since many foreign substances do not absorb or disappear on their own, surgical treatment is required. If blood-circulation disorders, tissue necrosis, or discoloration are found, a more invasive surgical approach may be required. In addition, esthetic or psychological concerns of the patient may also be indications for surgical procedures. Although it was difficult to completely remove foreign substances, we excised most of the material and generally attained satisfactory results in all cases. In addition, the results of this study confirmed that the treatment period could be shortened by relatively early surgical treatment.

An incision too small to achieve a good cosmetic results can make it difficult to approach and attain an adequate visual field. Therefore, extensive removal of areas within the symptomatic area, including skin and surrounding tissues of granuloma, is useful for correcting contours and resolving inflammation with high patient satisfaction.

Park et al. [19] introduced modifying perilesional approach by comparing the advantages and disadvantages of detoured approaches that consider cosmetic purposes with those of surgery through a direct incision. This method directly helped patients with severe foreign body granulomas, which are difficult 
to treat with ordinary nonsurgical procedures.

Choi et al. [20] described the usefulness of hydrosurgery in the surgical removal of foreign body granulomas. Although we did not adopt this method in our study, it seems to be a valuable treatment for managing patients with irregularities and deformities in a wide range of areas such as the forehead.

In the evaluation of patient satisfaction in this study, two patients complained of incomplete removal with symptoms of intermittent swelling. Complete removal was not possible due to extensive lesions on the forehead, leading to recurrent inflammation due to the foreign bodies remaining after surgery. Usually, in the case of foreign body granuloma, complete removal of those materials is problematic due to unclear margins. As such, materials can infiltrate the surrounding tissues, which is why foreign body granulomatous reactions may recur again in the future. Swelling of the periocular area after fat injection in the forehead has been reported and could suggest leakage of the injected fat [21]. It should be noted that for such patients, the treatment period may be longer and compliance may decrease. Before managing a foreign body reaction or granulomatous lesion, it is essential to consider these issues and obtain consent from the patient with sufficient explanation.

Although pathologic examination has little effect on the treatment method or outcome after treatment, the limitation of this study was that relatively few cases included pathologic findings. The pathological diagnosis of foreign body granulomas should be obtained through biopsy only. Also, it was not possible to definitively determine the type of fillers or injection materials used during illegal procedures. If the patient's status before and after treatment could be compared by radiological examination rather than the subjective assessments, the results of treatment could be evaluated more objectively. We suggested a treatment algorithm and confirmed patient symptoms and issues by establishing an accurate diagnosis. Prompt surgical treatment can shorten recovery time and lead to generally satisfactory treatment outcomes.

\section{NOTES}

\section{Conflict of interest}

No potential conflict of interest relevant to this article was reported.

\section{Ethical approval}

The study was approved by the Institutional Review Board of Kyung Hee University Hospital (IRB No. 2020-12-001) and performed in accordance with the principles of the Declaration of Helsinki. Written informed consent was obtained.

\section{Patient consent}

The patient provided written informed consent for the publication and use of their images.

\section{ORCID}

Jin Woo Jang

https://orcid.org/0000-0002-5073-8666

Sang Yoon Kang

https://orcid.org/0000-0002-6299-6371

\section{Author contribution}

Conceptualization: JWJ, SYK. Data curation: JWJ. Formal analysis, methodology: JWJ, SYK. Project administration: JWJ. Supervision: SYK. Writing - original draft: JWJ. Writing - review \& editing: JWJ, SYK.

\section{REFERENCES}

1. Ohashi M, Chiba A, Nakai H, Fukuda E, Higuchi T. serial injections of cryopreserved fat at $-196^{\circ} \mathrm{C}$ for tissue rejuvenation, scar treatment, and volume augmentation. Plast Reconstr Surg Glob Open 2018;6:e1742.

2. Engelman DE, Bloom B, Goldberg DJ. Dermal fillers: complications and informed consent. J Cosmet Laser Ther 2005;7:2932.

3. Williams GT, Williams WJ. Granulomatous inflammation: a review. J Clin Pathol 1983;36:723-33.

4. Lemperle G, Gauthier-Hazan N, Wolters M, Eisemann-Klein M, Zimmermann U, Duffy DM. Foreign body granulomas after all injectable dermal fillers: part 1 . Possible causes. Plast Reconstr Surg 2009;123:1842-63.

5. Lowe NJ, Maxwell CA, Patnaik R. Adverse reactions to dermal fillers: review. Dermatol Surg 2005;31(11 Pt 2):1616-25.

6. Jones DH, Carruthers A, Orentreich D, Brody HJ, Lai MY, Azen S, et al. Highly purified 1000-cSt silicone oil for treatment of human immunodeficiency virus-associated facial lipoatrophy: an open pilot trial. Dermatol Surg 2004;30:1279-86.

7. Park KG, Dhong ES, Namgoong S, Han JK, Han SK, Kim WK. Atypical facial filler granuloma: comparative histologic analysis with paraffinoma. Arch Craniofac Surg 2016;17:169-72.

8. Lee JM, Kim YJ. Foreign body granulomas after the use of dermal fillers: pathophysiology, clinical appearance, histologic features, and treatment. Arch Plast Surg 2015;42:232-9.

9. Kim CY. Overview of filler: compositions, effects, rheological consideration. J Korean Soc Aesthetic Plast Surg 2011;17:1-5.

10. Hong WT, Kim J, Kim SW. Minimizing tissue damage due to filler injection with systemic hyperbaric oxygen therapy. Arch Craniofac Surg 2019;20:246-50.

11. Coleman SR. Structural fat grafts: the ideal filler? Clin Plast Surg 2001;28:111-9. 
12. Hong KY. Fat grafts enriched with adipose-derived stem cells. Arch Craniofac Surg 2020;21:211-8.

13. Gutowski KA; ASPS Fat Graft Task Force. Current applications and safety of autologous fat grafts: a report of the ASPS fat graft task force. Plast Reconstr Surg 2009;124:272-80.

14. Guerrerosantos J, Gonzalez-Mendoza A, Masmela Y, Gonzalez MA, Deos M, Diaz P. Long-term survival of free fat grafts in muscle: an experimental study in rats. Aesthetic Plast Surg 1996;20:403-8.

15. Yang HJ, Kang SY. Comparisons between fresh and cryopreserved fat injections in facial lipofilling. Arch Craniofac Surg 2020;21:15-21.

16. Requena L, Requena C, Christensen L, Zimmermann US, Kutzner H, Cerroni L. Adverse reactions to injectable soft tissue fillers. J Am Acad Dermatol 2011;64:1-34.

17. Doh GH, Bahk S, Hong KY, Lim SA, Han KM, Eo SR. Delayed formation of sterile abscess after zygomaticomaxillary complex fracture treatment with bioabsorbable plates. Arch Craniofac Surg 2018;19:143-7.

18. Ko EY, Sung HM, Cho G, Park YK, Tak KS, Suh IS, et al. Usefulness of high-resolution ultrasonography after foreign body injection on aesthetic plastic surgery. J Korean Soc Plast Reconstr Surg 2010;37:385-90.

19. Park TH, Seo SW, Kim JK, Chang CH. The efficacy of perilesional surgical approach for foreign body granuloma. Plast Reconstr Surg 2011;127:121e-123e.

20. Choi M, Son KM, Choi WY, Cheon JS. Usefulness of the Versajet hydrosurgery system for the removal of foreign body granuloma. Arch Plast Surg 2017;44:352-3.

21. Park JW. Ocular swelling after forehead fat graft. Arch Aesthetic Plast Surg 2014;20:85-91. 